\title{
¿Son útiles los Antisecretores en el manejo de la Hemorragia Digestiva Alta no varicosa?
}

\author{
Joaquín Rodríguez Sánchez-Migallónª, Bartolomé López Viedmaa

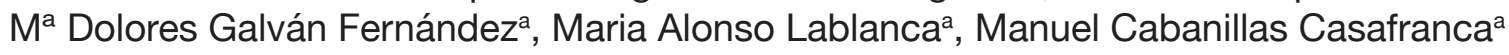 \\ Eduardo Rodríguez Sánchez ${ }^{a}$
}

\begin{abstract}
a Unidad de Medicina Digestiva, Hospital General de Ciudad Real, España.

Correspondencia: Joaquín Rodríguez Sánchez-Migallón, Unidad de Endoscopia (Pruebas especiales), Hospital General de Ciudad Real, C/ Obispo Rafael Torija s/n, Ciudad Real, España. Telf.: 638489515, correo electrónico: joakinrodriguez@gmail.com
\end{abstract}

Recibido el 26 de septiembre de 2009.

Aceptado para su publicación el 30 de septiembre de 2009.

\begin{abstract}
RESUMEN
La hemorragia digestiva alta no varicosa supone la causa más importante de sangrado digestivo, siendo una consulta frecuente en los servicios de urgencias hospitalarios. El tratamiento médico basado en el uso de inhibidores de la bomba de protones (IBP), junto con el tratamiento endoscópico, ha demostrado disminuir de manera significativa las tasas de resangrado, mejorando la supervivencia de estos pacientes. Después de dos décadas desde la comercialización de los (IBP), a día de hoy sigue existiendo controversia en cuanto a su dosificación y forma de administración en los pacientes con hemorragia digestiva de origen péptico.
\end{abstract}

Palabras clave: Úlcera Péptica. Inhibidores de la Bomba de Protones. Hemorragia Gastrointestinal.

\section{ABSTRACT}

Are anti-secretory drugs useful for the management of non-variceal upper gastrointestinal haemorrhage?

Non-variceal upper gastrointestinal haemorrhage, is the most frequent cause of gastrointestinal bleeding and is a common presentation in emergency departments. Medical treatment based on the use of proton pump inhibitors (PPIs), together with endoscopic therapy, has been shown to significantly lower rates of rebleeding and improve survival in these patients. Two decades after the marketing of PPIs there is still controversy as to dosage and method of administration for patients with gastrointestinal bleeding of peptic origin.

Key words. Peptic Ulcer, Proton Pump Inhibitors, Gastrointestinal Hemorrhage.

\section{INTRODUCCIÓN}

La hemorragia digestiva es una patología que plantea numerosos retos para el facultativo que se enfrenta a ella debido al amplio espectro clínico que abarca. Dependiendo de la región anatómica donde se origine el sangrado, toda hemorragia digestiva puede clasificarse en: alta (origen en la región proximal al ángulo de Treitz), baja (origen en región colónica), media (si tiene origen en intestino delgado), oculta (cuando es desconocida para el paciente) y de origen oscuro (de localización desconocida en el tubo digestivo). Dentro de todas ellas, la hemorragia digestiva alta no varicosa (HDANV) supone la causa principal de hemorragia digestiva, siendo cinco veces más frecuente que la que se origina en el tracto digestivo inferior. Se estima que la incidencia anual se encuentra en torno a 50-150 casos por 100.000 habitantes $^{1}$, debiéndose, algo más de la mitad de los casos, a la úlcera péptica gastroduodenal (tabla 1), de lo que se deduce que se trata de un motivo frecuente de consulta en los servicios de urgencias.

El tratamiento farmacológico de esta patología, lejos de ser un mero acompañante del tratamiento endoscópico, supone la piedra angular en el incremento de la supervivencia y la disminución en sus tasas de resangrado.

\section{BASES FISIOLÓGICAS DEL TRATAMIENTO CON ANTISECRETORES}

El estómago humano contiene alrededor de mil millones de células parietales, capaces de producir un $\mathrm{pH}$ intragástrico de 0,8 durante largos periodos de tiem$\mathrm{po}^{5}$. Esta producción de ácido se encuentra regulada por mediadores neurológicos, exocrinos y paracrinos. El mediador fundamental encargado de inhibir esta secreción es la somatostatina, la cual se libera una vez que los nutrientes alcanzan los primeros tramos del duodeno, para así conseguir el pH óptimo que capacite la absorción de sustancias a lo largo de todo el intestino delgado. Los 
mediadores más destacados en fomentar la secreción de ácido son la gastrina, liberada por las células $\mathrm{G}$ antrales al torrente circulatorio, la acetilcolina, liberada por las terminaciones vagales intramurales, la histamina, liberada por las células enterocromafines y mastocitos hacia el intersticio gástrico, y las más recientemente descubiertas grelina y orexina ${ }^{4}$, encargadas también de la regulación del apetito.

Cada uno de estos mediadores actúa sobre unos receptores específicos localizados en la membrana basolateral de la célula parietal. Los receptores colinérgicos (M3) y los receptores de gastrina (CCKb) usan como mediador el calcio citosólico, mientras que los receptores de histamina $(\mathrm{H} 2)$ incrementan los niveles de AMPc plasmático. Ambas vías confluyen en la activación de una bomba $\mathrm{H}+/ \mathrm{K}+-\mathrm{ATPasa}$, encargada de incrementar los niveles de protones en la luz gástrica para alcanzar el pH óptimo para la activación de la pepsina y que debe situarse por debajo de 2 .

De todo ello, además, se deduce que el ácido gástrico desempeña un papel crucial en la fisiopatología de las lesiones pépticas del esófago, estómago y duodeno, y que la inhibición adecuada de la secreción ácida facilita la cicatrización de lesiones a nivel de la mucosa digestiva. En 1990, Burget et $\mathrm{al}^{7}$ demostraron que la tasa de cicatrización de las úlceras duodenales es directamente proporcional al tiempo de supresión del $\mathrm{pH}$ gástrico en 24 horas, hecho fundamental para el desarrollo del concepto de inhibición ácida potente, que se define como aquélla que llevada a cabo es capaz de mantener un $\mathrm{pH}$ intragástrico por encima de 4 durante al menos 16 horas al día ${ }^{8}$, lo cual supone disminuir la concentración de $\mathrm{H}+$ en el jugo gástrico 1.000 veces por debajo de sus valores basales, hecho que ha demostrado disminuir la tasa de recurrencia en la úlcera gástricå .

Dentro del grupo de fármacos antisecretores, se dispone de 2 tipos: los anti-histamínicos (anti-H2) y los inhibidores de la bomba de protones (IBP), con notables diferencias en cuanto a la diana de actuación en la célula parietal, así como su efecto sobre la inhibición del $\mathrm{pH}$ intragástrico. Son diversos los estudios que han comparado la acción de los anti-H2 y los IBP sobre la secreción acida gástrica llegando a conclusiones similares. La potencia de los anti-H2 (famotidina $20 \mathrm{mg}$ y ranitidina 150 y $300 \mathrm{mg}$ ), en dosis única, es similar a la de un IBP, pero con efecto más rápi$\mathrm{do}^{9-11}$. Sin embargo, se produce una tolerancia a los anti-H2 que da lugar a un descenso de su capacidad antisecretora después de las primeras 12 horas de su administración, mientras que el efecto antisecretor de los IBP aumenta en función de las dosis repetidas del fármaco, alcanzando su máximo efecto a los 4-7 días de su administración (figura 1). Esto es debido a que el efecto de los anti-H2 se realiza en las fases iniciales de la vía de la secrección acida, provocando, si se administra de forma continua, un efecto de tolerancia en la célula parietal ${ }^{12}$. Por el contrario, la acción de los IBP tiene lugar al final de dicha vía, inhibiendo la acción de la bomba $\mathrm{H}+/ \mathrm{K}+-$ ATPasa de manera irreversible a través de un derivado sulfonamido, dando lugar al denominado complejo inhibitorio ${ }^{5}$, el cual obliga a la célula a sintetizar nuevas bombas ATPasa para mantener el $\mathrm{pH}$ en los límites fisiológicos $(\mathrm{pH}<2)$.

\section{IMPLICACIONES CLÍNICAS DE LA ACCIÓN DE LOS IBP}

Se ha estudiado cómo la pepsina es capaz de actuar como agente fibrinolítico sobre el coágulo de una úlcera péptica sangrante, lo que clínicamente se traduce en la imposibilidad de conseguir un control de la hemorragia, siempre y cuando el pepsinógeno se active en el jugo gástrico. Es aquí donde el concepto anteriormente desarrollado de inhibición ácida potente cobra mayor importancia. Estudios realizados "in vitro" demuestran cómo aumentando el pH gástrico

\begin{tabular}{l|c}
\hline Úlcera péptica gastroduodenal & $20-50 \%$ \\
Síndrome de Mallory-Weiss & $15-20 \%$ \\
Erosiones gástricas y duodenales & $10-15 \%$ \\
Esofagítis y úlceras esofágicas & $5-10 \%$ \\
Malformaciones vasculares & $5 \%$ \\
Neoplásias & $1 \%$ \\
Otras causas & $5 \%$ \\
\hline
\end{tabular}

Tabla 1. Etiología de la HDANV (modificado de Feu $\mathrm{F}$ et $\mathrm{al}^{3}$ ).

\begin{tabular}{llc}
\hline Estadio & Endoscopia & \% Resangrado \\
\hline IA & Sangrado activo a chorro & $55(17-100)$ \\
IB & Sangrado babeante & $55(17-100)$ \\
IIA & Vaso visible sin coágulo adherido & $43(35-55)$ \\
IIB & Coágulo adherido & $22(14-37)$ \\
IIC & Hematina & $7(5-10)$ \\
III & Tapón de fibrina & $2(0-5)$ \\
\hline
\end{tabular}

Tabla 2. Clasificación de Forrest (modificado de Forrest et al ${ }^{25}$ ). 


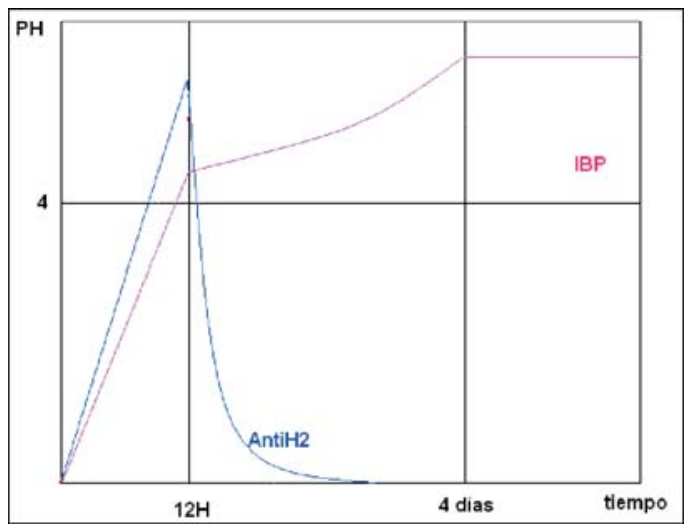

Fig. 1. Efecto comparativo de anti-H2/IBP.

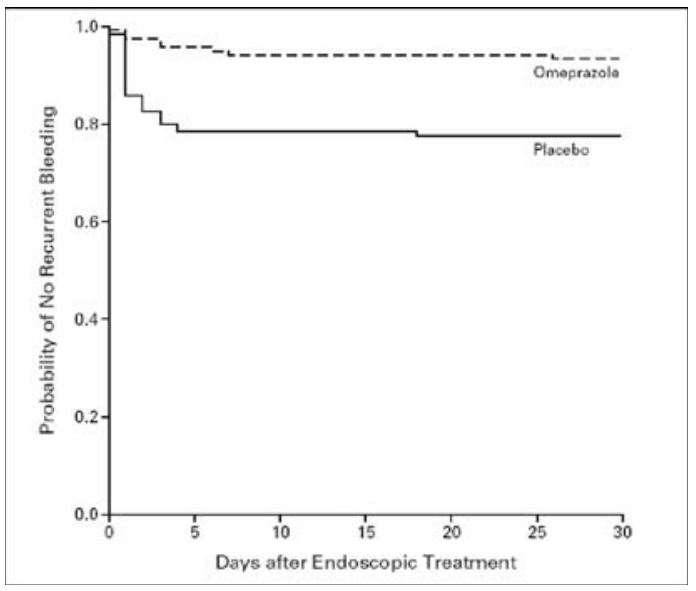

Fig. 2. Tasa de resangrado post-endoscopia (modificado de Lau et $\mathrm{al}^{21}$ )

por encima de 4 se posibilita la formación de puentes de fibrina, y por encima de 7 se consigue la estabilización del coágulo ${ }^{11-12}$. Para conseguir estas tasas de $\mathrm{pH}$ es necesario, debido a su distribución logarítmica, disminuir la concentración de $\mathrm{H}+$ en el jugo gástrico cerca de 1 millón de veces.

El citocromo P 2C19 es el enzima encargado del metabolismo de la mayoría de los IBP, condicionando así sus concentraciones plasmáticas. Existen diferentes mutaciones del gen que codifica el citocromo P 2C19, dando lugar a enzimas con distintos grados de actividad. Hay estudios que describen tres fenotipos diferentes: metabolizadores rápidos (MR), metabolizadotes lentos (ML) e individuos con alelo mutante. Cuando se somete a estos individuos a la acción de distintos IBP (omeprazol, lansoprazol y rabeprazol) se observa ${ }^{14}$ que el $\mathrm{pH}$ intragástrico en los ML alcanza valores promedio de 6 , mientras que en los MR tan sólo alcanza cifras en torno a 3. Por lo tanto, este efecto sobre la concentración plasmática del fármaco condiciona notablemente la respuesta al tratamiento. Es curioso observar cómo en estudios comparativos de IBP vs. antiH2 o placebo se obtienen mejores resultados en cuanto a tasas de resangrado y mortalidad en países asiáticos, lo que podría ser debido a que la tasa de metabolizadores lentos es mucho más frecuente en la población asiática que en la caucásica.

Recientemente se ha publicado un estudio donde a través de un test de medición de [C13] en aire exhalado se determina la actividad del citocromo P 2C19 mediante la administración de [C13]-pantoprazol, lo cual supone una importante aplicación clínica, ya que podemos predecir la respuesta al IBP y por lo tanto individualizar la dosis de tratamiento ${ }^{18}$.

\section{IBP ANTES DE LA ENDOSCOPIA}

Parece pues lógico que una vez diagnosticada una hemorragia digestiva alta, habiendo excluido previamente a los pacientes con alta sospecha de hemorragia por hipertensión portal, se inicie tratamiento con IBP a la espera de llevar a cabo la valoración endoscópica. En base a esto, en el año 2006 se publicó en Cochrane Library un meta-análisis ${ }^{19}$ que incluía 1.512 pacientes en los que se analizaron los efectos de la administración de IBP previo al tratamiento endoscópico. Este estudio llegó a la conclusión de que el tratamiento pre-endoscópico con IBP en la HDANV no disminuye la tasa de resangrado ni la mortalidad, pero sí encontró una reducción significativa en la proporción de pacientes con hemorragia activa, vaso visible no sangrante o coágulo adherido, en el momento de realizar la endoscopia ( $46 \%$ en no tratados frente al $36 \%$ en tratados). En conclusión, podemos decir que la administración de IBP antes de la endoscopia no reduce la mortalidad ni la tasa de resangrado, pero si mejora los hallazgos endoscópicos basándose en la clasificación de Forrest ${ }^{24}$ (tabla 2).

\section{IBP DESPUÉS DE LA ENDOSCOPIA.}

La terapéutica endoscópica consigue un control de la hemorragia en aproximadamente el $90 \%$ de los pacientes, con unas tasas de resangrado en torno al $10-30 \%$ de los casos $^{20}$, con gran repercusión sobre el pronóstico de la enfermedad. Es aquí donde el papel de los IBP cobra mayor importancia. En el año 2000 Lau et al ${ }^{21}$ publicaron un estudio donde se concluyó que la infusión de omeprazol, $8 \mathrm{mg} / \mathrm{h}$ durante las primeras 72 horas post-endoscopia, disminuye de manera significativa las tasas de resangrado (figura 2). Cinco años más tarde se publicó un meta-análisis ${ }^{22}$ que llegó a la conclusión de que el efecto beneficioso de los IBP después de la endoscopia es independiente de la vía de administración y de la dosis, dato concordante con un estudio publicado recientemente por G. Javit et al ${ }^{23}$, en el que se randomizó a una cohorte de 90 pacientes a recibir omeprazol, pantoprazol y 
lansoprazol por vía oral e intravenosa a altas dosis, durante las primeras 72 horas post-endoscopia. La conclusión a la que se llegó en este estudio es que no existen diferencias entre los distintos IBP y su vía de administración, en cuanto al rango de $\mathrm{pH}$ alcanzado ( $\mathrm{pH}$ de 6 durante las primeras 72 horas). Pero en la recidiva hemorrágica no sólo influye el hecho de utilizar IBP. Cheng et $\mathrm{al}^{24}$ demostraron que la administración de omeprazol a bajas dosis $(3,3 \mathrm{mg} / \mathrm{h})$, durante los primeros 7 días post-endoscopia, disminuye la recidiva hemorrágica durante los primeros 28 días, en comparación con los que reciben la dosis habitual $(8 \mathrm{mg} / \mathrm{h})$ durante 3 días, pero, además, se pone de manifiesto la importancia de la comorbilidad en la posibilidad de resangrado, dado que se observa también que el hecho de tener una albúmina sérica < $3 \mathrm{~g} / \mathrm{dl}$ o un tumor diseminado actúan como factores independientes de recidiva hemorrágica.

De este modo, es probable que en un futuro, dependiendo de las características de cada paciente, se apliquen pautas individualizadas de tratamiento, variando las dosis y pauta de administración.

\section{CONCLUSIONES}

La HDANV supone la causa más frecuente de sangrado digestivo, siendo una consulta frecuente en los servicios de urgencias hospitalarios, con una incidencia anual de 50-150 casos/100.000 habitantes. El tratamiento farmacológico basado en el uso de IBP juega un papel fundamental en el manejo de esta patología. Aunque no existen evidencias de que su administración previa a la endoscopia mejore las tasas de resangrado y la mortalidad, sí se ha demostrado que su administración mejora los hallazgos endoscópicos (hecho que sí está directamente relacionado con las tasas de resangrado y morbi/mortalidad).

El uso de IBP después de la endoscopia ha demostrado ser claramente beneficioso, reduciendo de manera significativa la tasa de resangrado, así como la necesidad de cirugía. Sin embargo, son necesarios más estudios prospectivos para contrastar la vía y el tiempo de administración más adecuado de los IBP para, en función de las características de comorbilidad de cada paciente, poder diseñar pautas terapéuticas "a la carta".

\section{BIBLIOGRAFÍA}

1. Laine L, Peterson WL. Bleeding peptic ulcer. N Engl J Med 1994; 331:717-27.

2. Pardo $A$, Durández $R$, Hernández $M$ et al. Impact of physician specialty on the cost of nonvariceal upper Gl bleeding care. Am J Gastroenterol 2002; 97:1535-42.

3. Feu F, Brullet E, Calvet $X$ et al. Recomendaciones para el diagnóstico y tratamiento de la hemorragia digestiva alta agudo no varicosa. Gastroenterol Hepatol 2003; 26(2):70-85.
4. Schubert ML .Gastric exocrine and endocrine secretion. Curr Opin Gastroenterol 2009 (article in press).

5. Esplugues JV. A pharmacological approach to gastric acid inhibition. Drugs 2005; 65 (suppl 1):7-12.

6. Koelz HR Gastric acid in vertebrates.Scand J Gastroenterol Suppl 1992; 193:2-6.

7. Burget DW, Chiverton SG, Hunt RH et al. Is there an optimal degree of acid suppression for healing of duodenal ulcers? A model of the relationship between ulcer healing and acid suppression. Gastroenterology 1990; 99(2):345-51.

8. Gomollón F, Calvet X. Optimising acid inhibition treatment. Drugs 2005; 65 (Suppl 1):25-33.

9. lida $\mathrm{H}$, Inamori $\mathrm{M}$, Akimoto $\mathrm{K}$ et al. Early effects of intravenous administrations of lansoprazole and famotidine on intragastric pH. Hepatogastroenterology 2009; 56(90):551-4.

10. Abe $\mathrm{Y}$, Inamori M, Togawa J et al. The comparative effects of single intravenous doses of omeprazole and famotidine on intragastric pH. J Gastroenterol 2004; 39(1):21-5.

11. Hedenström $\mathrm{H}$, Alm $\mathrm{C}$, Kraft $\mathrm{M}$ et al. Intragastric $\mathrm{pH}$ after oral administration of single doses of ranitidine effervescent tablets, omeprazole capsules and famotidine fast-dissolving tablets to fasting healthy volunteers. Aliment Pharmacol Ther 1997; 11(6):1137-41.

12. Sachs G. Physiology of the parietal cell and therapeutic implications. Pharmacotherapy 2003; 23(10 Pt 2):68S-73S.

13. Gacko M, Roszkowska-Jakimiec W, Jasielczuk J. et al. Anticoagulative effect of pepsin. Acta Biochim Pol 1999; 46(3):717-20.

14. Patchett SE, Enright $\mathrm{H}$, Afdhal $\mathrm{N}$ et al. Clot lysis by gastric juice: an in vitro study. Gut 1989; 30:1704-7.

15. Bardou M, Martín J, Barkun A et al. Intravenous proton pump inhibitors: an evidence-based review of their use in gastrointestinal disorders. Drugs 2009; 69(4):435-48.

16. Leontiadis GI, Sharma VK, Howden CW. Proton Pump Inhibitor Therapy for Peptic Ulcer Bleeding: Cochrane Collaboration Meta-analysis of Randomized Controlled Trials. Mayo Clin Proc 2007; 82(3):286-296.

17. Klotz U. Clinical impact of CYP2C19 polymorphism on the action of proton pump inhibitors: a review of a special problem. Int J Clin Pharmacol Ther 2006; 44(7):297-302.

18. Furuta T, Kodaira $\mathrm{C}$, Nishino $\mathrm{M}$ et al. [13C]-pantoprazole breath test to predict CYP2C19 phenotype and efficacy of a proton pump inhibitor, lansoprazole. Aliment Pharmacol Ther 2009; 30(3):294-300.

19. Dorward S, Sreedharan A, Leontiadis GI et al. Proton pump inhibitor treatment initiated prior to endoscopic diagnosis in upper gastrointestinal bleeding. Cochrane Database Syst Rev 2006; (4):CD005415.

20. Rockall TA, Logan R F, Devlin HB et al. Risk assessment after acute upper gastrointestinal haemorrhage. Gut 1996; 38:31621

21. Lau JYW, Sung JJY, Lee KC et al. Effect of intravenous Omeprazole on recurrent bleeding after endoscopic treatment of bleeding peptic ulcers. N Engl J Med 2000; 343:310-16.

22. Andrivlli $\mathrm{A}$, Aneese $\mathrm{V}$, Caruso $\mathrm{N}$ et al. Proton pump inhibitors and outcome of endoscopic hemostasis in bleeding peptic ulcer. A series of meta analysis. Am J Gastroenterol 2005; 100:207-19.

23. Javid G, Zargar SA, U-Saif R et al. Comparison of p.o. or i.v. proton pump inhibitors on 72-h intragastric $\mathrm{pH}$ in bleeding peptic ulcer. J Gastroenterol Hepatol 2009; 24:1236-43.

24. Cheng H, Chang WL, Yeh YC et al. Seven-day intravenous lowdose omeprazole infusion reduces peptic ulcer rebleeding for patients with comorbidities. Gastrointest Endosc 2009 (article in press).

25. Forrest JAH, Finlayson NDC, Shearman DJC. Endoscopy in gastrointestinal bleeding. Lancet 1974; 2:394-7. 